\title{
Influência da Composição Corporal sobre a Qualidade de Vida de Pacientes com Câncer
}

\author{
The Influence of Body Composition on Cancer Patients' Quality of Life \\ Influencia de la Composición Corporal sobre la Calidad de Vida de Pacientes \\ con Cáncer
}

Camila Conde Frio ${ }^{1}$; Alessandra Doumid Borges Pretto ${ }^{2}$; Maria Cristina Gonzalez ${ }^{3}$; Carla Alberici Pastore ${ }^{4}$

\section{Resumo}

Introduçáo: $\mathrm{O}$ câncer tem reflexos na composição corporal, frequentemente causando sarcopenia, o que impacta na capacidade funcional e prognóstico dos doentes, podendo afetar sua qualidade de vida. Objetivo: Avaliar a influência da composição corporal sobre a qualidade de vida de pacientes com câncer de trato gastrointestinal e de pulmão. Método: Estudo transversal com pacientes portadores de câncer gastrointestinal e de pulmão, no serviço de quimioterapia do Hospital Escola da Universidade Federal de Pelotas. A composição corporal foi estimada por meio da bioimpedância elétrica e da qualidade de vida por meio do instrumento European Organization for Research and Treatment of Cancer - Quality of Life Questionnaire Core-30. Resultados: Foram avaliados 74 pacientes, sendo a maioria do sexo masculino $(56,8 \%)$. Os tumores mais prevalentes foram os de trato gastrointestinal $(75,4 \%)$. Aproximadamente $24 \%$ dos pacientes apresentaram déficit de massa muscular e nenhum apresentou excesso de adiposidade. $\mathrm{Na}$ análise da relação entre composição corporal e as diferentes escalas de qualidade de vida, os pacientes com déficit de massa muscular apresentaram escores mais baixos nas escalas de Saúde Geral/QV e funcional, e maiores pontuaçóes na escala de sintomas, demonstrando pior qualidade de vida quando comparados aqueles sem déficit de massa muscular. Conclusão: A composição corporal com déficit de massa magra se associou à pior Qualidade de Vida em pacientes com câncer de trato gastrointestinal e de pulmão.

Palavras-chave: Neoplasias Pulmonares; Neoplasias Gastrointestinais; Composição Corporal; Qualidade de Vida

Faculdade de Nutrição da Universidade Federal de Pelotas (UFPel). Pelotas (RS), Brasil.

${ }^{1}$ Nutricionista pela Faculdade de Nutriçáo da UFPel. Pelotas (RS), Brasil. E-mail: camila.sconde@yahoo.com.br.

${ }^{2}$ Nutricionista. Doutora em Saúde e Comportamento pela Universidade Católica de Pelotas (UCPel). Pelotas (RS), Brasil. E-mail: alidoumid@yahoo.com.br.

${ }^{3}$ Médica. Doutora em Epidemiologia. Professora-titular do Programa de Pós-Graduaçáo em Saúde e Comportamento da UCPel. Pelotas (RS), Brasil.

E-mail: mcgonzalez@hotmail.com.

${ }^{4}$ Nutricionista. Doutora em Saúde e Comportamento pela UCPel. Pelotas (RS), Brasil. E-mail: pastorecarla@yahoo.com.br.

Endereşo para correspondência: Carla Alberici Pastore. Rua Taquari, 617 - Laranjal. Pelotas (RS), Brasil. CEP: 96090-770. E-mail: pastorecarla@yahoo.com.br. 


\section{INTRODUÇÃO}

As neoplasias malignas representam um problema de saúde pública mundial, sendo definidas pelo crescimento celular anormal, descoordenado e sem finalidade útil ao organismo, que ocorrem pela interaçấo de fatores internos (genéticos) e externos (estilo de vida, relacionado a hábitos) ${ }^{1}$. Segundo estimativas da Organização Mundial da Saúde (OMS), em 2030, surgirão 27 milhôes de novos casos de câncer; 17 milhôes de mortes serão por câncer; e haverá 75 milhóes de pessoas vivas com a doença. No Brasil, o câncer se configura como problema de saúde pública, uma vez que as neoplasias ganharam importância crescente no perfil de mortalidade do país, ocupando o segundo lugar como causa de óbito ${ }^{2}$.

Sabe-se que o câncer tem reflexos na composição corporal do doente oncológico, tendo como característica a perda de massa magra com uma preservação relativa de massa proteica visceral ${ }^{1}$. A sarcopenia, encontrada em pacientes oncológicos, definida como perda de massa muscular independente de perda de tecido adiposo, tem sido associada à diminuição da capacidade funcional, ao aumento do risco de quedas e fraturas, à maior tempo de hospitalização e ao aumento de infecçôes nosocomiais ${ }^{3}$.

Outra consequência comum aos pacientes com câncer é a desnutrição, que pode avançar para um quadro de caquexia, levando desde a perda de peso até a uma incapacidade geral que pode progredir para morte. Em estudo com pacientes com câncer de pulmão de não pequenas células, verificou-se que $35,7 \%$ dos doentes analisados apresentavam desnutriçáo e isso estava relacionado a sintomas como falta de apetite, fadiga, náuseas, vômitos e obstipação, resultando em pior qualidade de vida $(\mathrm{QV})$ nesses doentes comparados com aqueles que não apresentavam desnutrição ${ }^{4}$.

A perda ponderal involuntária, diminuição da capacidade funcional, depleção progressiva de massa magra e tecido adiposo caracterizam a caquexia, condição de desnutrição energético-proteica grave. Essa condição afeta a capacidade funcional, a resposta ao tratamento, a QV e a sobrevida do paciente 5 .

Segundo a OMS, QV é definida como a forma que um indivíduo percebe sua posição na vida, suas expectativas, $\mathrm{o}$ contexto cultural e o sistema de valores que experimenta em relação aos seus objetivos, aos padrōes e às preocupaçôes ${ }^{6}$. Diante da magnitude do câncer e da possibilidade de alguns pacientes evoluírem para estágios terapeuticamente incontroláveis, é necessário identificar fatores que possam estar associados à melhora ou piora de sua $\mathrm{QV}^{7}$. No paciente oncológico, a QV é influenciada pela nutrição e composição corporal $^{1}$. Por isso, além de avaliar a QV do paciente com câncer, a avaliação da composição corporal se faz essencial, pois a perda de massa magra está diretamente associada à redução da capacidade funcional, entre outros aspectos negativos referentes à $\mathrm{QV}$ desses pacientes ${ }^{1,8}$.

Assim, este estudo tem por objetivo avaliar a influência da composição corporal sobre a QV de pacientes com câncer de trato gastrointestinal e de pulmão.

\section{MÉTODO}

Estudo transversal alinhado a uma coorte composta por 77 pacientes com câncer de trato gastrointestinal e de pulmão. A coleta de dados foi realizada antes do início da sua primeira seção de quimioterapia. A pesquisa original foi desenvolvida no Serviço de Quimioterapia do Hospital Escola da Universidade Federal de Pelotas (UFPel), no período de junho de 2008 a maio de 2010.

Os critérios de inclusão foram: ser maior de 18 anos de idade, ter câncer de trato gastrointestinal e de pulmão, com indicação de quimioterapia. Os pacientes com esse perfil foram convidados a participar do estudo durante a primeira consulta médica com indicação quimioterápica. Aqueles que aceitaram participar da pesquisa assinaram o Termo de Consentimento Livre e Esclarecido e foram encaminhados à consulta com um nutricionista previamente treinado para a coleta de dados. Os critérios de exclusão foram tratamento quimioterápico já iniciado, não ter indicação de quimioterapia e ser portador de prótese metálica ou marca-passo, devido à realizaçáo da bioimpedância.

As informaçóes demográficas e socioeconômicas foram obtidas por questionários padronizados. Também foi realizada coleta de dados, do sistema hospitalar informatizado, sobre a localizaçáo e o estadiamento do tumor, bem como o objetivo do tratamento quimioterápico. As medidas de peso e altura foram aferidas em balança antropométrica Filizola digital modelo PL 150, com capacidade de $150 \mathrm{~kg}$ e precisão de $100 \mathrm{~g}$ e estadiômetro de alumínio acoplado à balança com capacidade para 2,0 m e precisão de $5 \mathrm{~mm}$. Para classificar o estado nutricional dos pacientes, utilizou-se o Índice de Massa Corporal (IMC= peso $(\mathrm{Kg}) /$ altura $\left.(\mathrm{m})^{2}\right)$. Os pacientes foram também submetidos à Avaliação Subjetiva Global Produzida pelo Paciente (ASG-PPP) 9

A composição corporal foi estimada por meio da bioimpedância elétrica (BIA), realizada com instrumento BIA (RJL Systems ${ }^{\oplus}$ ). O exame foi realizado com os pacientes em jejum de oito horas e com a bexiga vazia. Para a classificação da composiçáo corporal, foram calculados, a partir dos dados da bioimpedância, o Índice de Massa Livre de Gordura (IMLG=peso da massa magra $(\mathrm{Kg}) /$ altura $(\mathrm{m})^{2}$ ) e o Índice de Massa de Gordura (IMG=peso da massa gorda $(\mathrm{Kg})$ /altura $\left.(\mathrm{m})^{2}\right)$. Estes foram avaliados 
de acordo com os pontos de corte sugeridos por Kyle et al. ${ }^{10}$ que classificam a composição corporal em déficit de massa muscular (homens IMLG $\leq 17,4 \mathrm{Kg} / \mathrm{m}^{2}$ e mulheres IMLG $\leq 15,0 \mathrm{Kg} / \mathrm{m}^{2}$ ) e excesso de adiposidade (homens $\mathrm{IMG} \geq 8,3 \mathrm{Kg} / \mathrm{m}^{2}$ e mulheres IMG $\geq 11,8 \mathrm{Kg} / \mathrm{m}^{2}$ ).

A avaliação da QV foi realizada por meio do instrumento European Organization for Research and Treatment of Cancer - Quality of Life Questionnaire Core30 (EORTC QLQ-C30), validado para a população brasileira. Esse instrumento possui 30 questóes, sendo composto por cinco escalas funcionais (funçôes física, cognitiva, social, emocional e desempenho de papel), três escalas de sintomas (fadiga, dor e náusea e vômito), uma escala de estado geral de saúde/QV e cinco itens que avaliam sintomas relatados por doentes com câncer: dispneia, perda de apetite, insônia, constipação e diarreia; além de um item de avaliação de impacto financeiro do tratamento e da doença. Os resultados são obtidos com valores nas escalas de funçôes física, cognitiva, social, emocional, desempenho de papel e estado geral de saúde/ $\mathrm{QV}$, sendo que quanto mais próximo de 100 melhor a QV.

Os dados foram digitados no software EpiInfo $6.04 \mathrm{~d}^{\oplus}$ e as análises estatísticas foram realizadas pelo pacote estatístico Stata ${ }^{\circledR}$ 11.1. Os dados categóricos tiveram suas frequências percentuais apresentadas e as contínuas, sua média e desvio-padrão - se paramétricos - ou mediana e intervalo interquartil (IIQ) - se não paramétricos. Para comparação de médias/medianas dos escores de QV em relação à presença de déficit de massa magra, foi utilizado o Teste $t$ de Student com nível de significância de 5\%. A pesquisa foi aprovada pelo Comitê de Ética da Faculdade de Medicina da UFPel, segundo ofício 066/06, de 30 de julho de 2006, de acordo com a Declaração de Helsinky (2000) e com a Resolução CNS 196/96.

\section{RESULTADOS}

A amostra do estudo original foi composta por 77 pacientes. Para este estudo, foi feita a exclusão de três pacientes que não realizaram o exame de BIA devido à não apresentação em jejum, contando ao final com 74 pacientes com câncer de trato gastrointestinal e de pulmão.

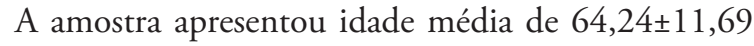
anos, sendo a maioria $(56,76 \%)$ do sexo masculino. A maior parte dos pacientes se declarou com cor de pele branca $(86,49 \%)$ e era casado ou vivia com companheiro (58,11\%). Quanto ao perfil socioeconômico, 60,81\% da amostra se enquadrava na classe $\mathrm{C}$, segundo classificação da Associação de Empresas de Pesquisa (ABEP 2013).

$\mathrm{O}$ sítio tumoral mais prevalente foi cólon e reto $(47,30 \%)$, seguido de pulmão $(24,32 \%)$, esôfago e estômago $(22,97 \%)$ e pâncreas e vesícula biliar $(5,41 \%)$.
Verificou-se que mais da metade dos pacientes tinha a doença em estadiamentos III e IV (60,56\%) e recebeu indicação de quimioterapia em caráter paliativo (52,70\%). A descrição detalhada da amostra está demonstrada na Tabela 1.

$\mathrm{O}$ estado nutricional avaliado pelo IMC apontou $8,11 \%$ dos pacientes com baixo peso (IMC $\leq 18,5 \mathrm{Kg} /$ $\left.\mathrm{m}^{2}\right)$; a maioria da amostra $(60,81 \%)$ foi classificada como eutrófica (IMC 18,5-24,9 Kg/m²); 29,73\% dos pacientes apresentaram sobrepeso (IMC $25-29,9 \mathrm{Kg} / \mathrm{m}^{2}$ ) e $1,35 \%$ estavam obesos $\left(\mathrm{IMC} \geq 30 \mathrm{Kg} / \mathrm{m}^{2}\right)$. Já a avaliação do estado nutricional segundo a ASG-PPP demonstrou que 68,92\% dos pacientes apresentavam desnutrição suspeita ou moderada, $18,92 \%$ desnutrição grave e $12,16 \%$ estavam bem nutridos.

Verificou-se, na análise da composição corporal, que a amostra geral apresentou média de massa livre de gordura de 47,05 $\pm 10,03 \mathrm{Kg}(77,71 \%)$ e média de massa

Tabela 1. Descrição da amostra de pacientes com câncer de trato gastrointestinal e de pulmão atendidos no Serviço de Quimioterapia do Hospital Escola da Universidade Federal de Pelotas (RS), Brasil 2008-2010

\begin{tabular}{|c|c|c|}
\hline Variável & $\mathbf{n}$ & $\%$ \\
\hline $\begin{array}{l}\text { Sexo } \\
\text { Masculino } \\
\text { Feminino }\end{array}$ & $\begin{array}{l}42 \\
32\end{array}$ & $\begin{array}{l}56,76 \\
43,24\end{array}$ \\
\hline $\begin{array}{l}\text { Cor (autorreferida) } \\
\text { Brancos } \\
\text { Não brancos }\end{array}$ & $\begin{array}{l}64 \\
10\end{array}$ & $\begin{array}{l}86,49 \\
13,51\end{array}$ \\
\hline $\begin{array}{l}\text { Estado civil } \\
\text { Sem companheiro } \\
\text { Com companheiro/casado }\end{array}$ & $\begin{array}{l}31 \\
43\end{array}$ & $\begin{array}{l}41,89 \\
58,11\end{array}$ \\
\hline $\begin{array}{l}\text { Perfil socioeconômico } \\
\text { A } \\
\text { B } \\
\text { C } \\
\text { D } \\
\text { E }\end{array}$ & $\begin{array}{c}0 \\
5 \\
45 \\
23 \\
1\end{array}$ & $\begin{array}{c}0,00 \\
6,76 \\
60,81 \\
31,08 \\
1,35\end{array}$ \\
\hline $\begin{array}{l}\text { Sítio tumoral } \\
\text { Esôfago/estômago } \\
\text { Cólon e reto } \\
\text { Pâncreas e vesícula biliar } \\
\text { Pulmão }\end{array}$ & $\begin{array}{c}17 \\
35 \\
4 \\
18\end{array}$ & $\begin{array}{c}22,97 \\
47,30 \\
5,41 \\
24,32\end{array}$ \\
\hline $\begin{array}{l}\text { Estadiamento do tumor } \\
\text { II } \\
\text { III } \\
\text { IV } \\
\text { Desconhecido }\end{array}$ & $\begin{array}{l}17 \\
25 \\
18 \\
11\end{array}$ & $\begin{array}{l}23,94 \\
35,21 \\
25,35 \\
15,49\end{array}$ \\
\hline $\begin{array}{l}\text { Indicação quimioterápica } \\
\text { Indeterminada } \\
\text { Curativa } \\
\text { Prévia/neoadjuvante } \\
\text { Adjuvante } \\
\text { Paliativa }\end{array}$ & $\begin{array}{c}1 \\
1 \\
22 \\
11 \\
39\end{array}$ & $\begin{array}{c}1,35 \\
1,35 \\
29,73 \\
14,86 \\
52,70\end{array}$ \\
\hline Total & 74 & 100,00 \\
\hline
\end{tabular}


de gordura de $13,80 \pm 5,60 \mathrm{Kg}(22,28 \%)$. A quantidade de água corporal da amostra foi de $56,89 \pm 5,36 \mathrm{Kg}$, enquanto a massa celular corporal apresentou média de $20,97 \pm 6,06$ Kg. A composição corporal percentual por sexo está demonstrada na Figura 1.

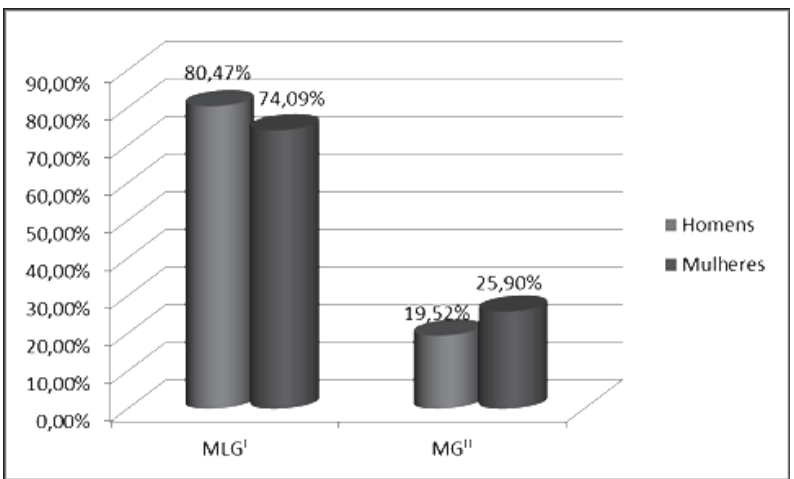

Figura 1. Composição corporal segundo o gênero de pacientes com câncer de trato gastrointestinal e de pulmão atendidos no Serviço de Quimioterapia do Hospital Escola da Universidade Federal de Pelotas (RS), Brasil - 2008-2010

${ }^{1}$ MLG- Massa Livre de Gordura.

${ }^{11}$ MG-Massa de Gordura.

A avaliação da composição corporal segundo os critérios de Kyle et al. ${ }^{10}$ mostrou que $24,3 \%$ da amostra apresentou déficit de massa muscular. Entre os homens, $23,81 \%$ apresentaram esse déficit (IMLG $\leq 17,4 \mathrm{Kg}$ / $\mathrm{m}^{2}$ ), enquanto entre as mulheres $25 \%$ tinham déficit de massa muscular (IMLG $\leq 15,0 \mathrm{Kg} / \mathrm{m}^{2}$ ). Nenhum paciente apresentou excesso de adiposidade (homens IMG $\geq 8,3$ $\mathrm{Kg} / \mathrm{m}^{2}$ e mulheres IMG $\geq 11,8 \mathrm{Kg} / \mathrm{m}^{2}$ ).

$\mathrm{Na}$ avaliação da QV, a escala de estado geral de saúde/ QV atingiu um escore médio de $67,11 \pm 22,91$ pontos, a escala funcional atingiu $69,42 \pm 22,72$ pontos e na escala de sintomas a mediana foi de 19,2 (IIQ 5,12; 43,6) pontos.

$\mathrm{Na}$ análise da relação entre composição corporal e as diferentes escalas de $\mathrm{QV}$, os pacientes com déficit de massa muscular apresentaram escores mais baixos nas escalas de Saúde Geral/QV e funcional, e maiores pontuaçóes na escala de sintomas, demonstrando pior QV quando comparados aqueles sem déficit de massa muscular, conforme observado na Tabela 2 .

\section{DISCUSSÃO}

Quando avaliado o estado nutricional segundo o IMC, a maior parte da amostra foi classificada como eutrófica, seguida de sobrepeso, e menos de $9 \%$ apresentaram baixo peso. Resultado diferente do encontrado em outros estudos com pacientes avaliados antes dos tratamentos cirúrgico e quimioterápico.
Tabela 2. Escores nas escalas de QV de acordo com a presença de déficit de massa muscular de pacientes com câncer de trato gastrointestinal e de pulmão atendidos no Serviço de Quimioterapia do Hospital Escola da Universidade Federal de Pelotas (RS), Brasil 2008-2010

\begin{tabular}{c|c|c|c}
\hline $\begin{array}{c}\text { Qualidade } \\
\text { de vida }\end{array}$ & \multicolumn{2}{|c|}{ Déficit de massa muscular } & \multirow{2}{*}{ p* } \\
$\begin{array}{c}\text { Escala } \\
\text { avaliada }\end{array}$ & $\begin{array}{c}\text { Sim } \\
\text { Média (DP) }\end{array}$ & $\begin{array}{c}\text { Não } \\
\text { Média (DP) }\end{array}$ & \\
\hline $\begin{array}{c}\text { Estado de } \\
\text { saúde geral/ } \\
\text { QV }\end{array}$ & $57,40 \pm 28,56$ & $70,23 \pm 20,09$ & 0,040 \\
\hline Funcional & $60,12 \pm 21,18$ & $72,42 \pm 22,57$ & 0,040 \\
\hline Sintomas & $37,46 \pm 21,40$ & $21,74 \pm 20,18$ & 0,006 \\
\hline
\end{tabular}

*Teste t de Student.

Estudo conduzido por Ferraz e Campos com pacientes com câncer gástrico encontrou $40 \%$ dos pacientes com peso adequado, $14 \%$ com sobrepeso e $46,0 \%$ apresentaram desnutriçấo ${ }^{11}$. Resultado semelhante foi encontrado em estudo, conduzido por Oliveira et al. ${ }^{12}$, com pacientes com neoplasia de cavidade oral, com maior prevalência (34,5\%) de baixo peso.

De acordo com a ASG-PPP, houve alta prevalência de desnutrição suspeita ou moderada (quase $70 \%$ da amostra), resultado semelhante ao encontrado em estudo com pacientes com carcinoma esofágico, em que $70 \%$ da populaçáa apresentaram desnutrição moderada ${ }^{13}$. Outro estudo realizado por Khoshnevis et al. ${ }^{14}$ com 416 pacientes com câncer encontrou uma prevalência de desnutrição em $53,1 \%$ da amostra, sendo $29,1 \%$ moderada e $24 \%$ desnutrição grave. Campos et al. ${ }^{15}$ mostraram que o número de indivíduos classificados como desnutridos $(45,1 \%)$ foi maior quando se empregou a ASG-PPP do que quando se utiliza o IMC.

No presente estudo, a amostra apresentou composiçấo corporal com cerca de $80 \%$ de massa livre de gordura. Trutschnigg et al. ${ }^{16}$ encontraram em sua amostra de pacientes com câncer avançado de pulmão de não pequenas células e neoplasia gastrointestinal, utilizando a BIA, uma quantidade de $75,22 \%$ de massa livre de gordura e $24,78 \%$ de gordura corporal. Em estudo realizado com pacientes desnutridos portadores de neoplasia e de doenças benignas do trato digestório, foram encontrados, na composiçáo corporal dos pacientes com câncer, $95,1 \%$ de massa magra e 4,9\% de gordura corporal ${ }^{17}$.

As escalas de QV avaliadas neste estudo apontam resultados semelhantes aos apresentados por outros trabalhos realizados com pacientes oncológicos e que utilizaram o EORTC QLQ-C30 como instrumento de avaliação, sendo assim demonstra estar dentro da variação de valores médios encontrados na literatura. Oliveira et al. ${ }^{18}$, ao avaliarem a QV de pacientes com câncer 
de pulmão, antes e após o tratamento quimioterápico, demonstraram que os pacientes após o tratamento apresentaram melhora da QV relacionada à saúde e aos sintomas do câncer de pulmáo, e piora dos sintomas decorrentes da toxicidade do tratamento. Em estudo realizado com pacientes norte-americanos com câncer colorretal, a média na escala de saúde geral/QV foi de 62,2 pontos; na escala funcional, o domínio que apresentou menor valor foi o de desempenho de papel $(68,4$ pontos); e na escala de sintomas fadiga (37,7 pontos) e insônia ( 35,1 pontos), foram os sintomas mais prevalentes na amostra ${ }^{19}$.

Também foram encontrados na literatura escores mais baixos do que os apresentados neste estudo na escala de saúde geral/QV, como em trabalho realizado na Malásia ${ }^{20}$, onde o escore médio nessa escala foi de 51 pontos; e 46,2 pontos em estudo de Trabal et al. ${ }^{21}$ com pacientes oncológicos realizado em um hospital de Barcelona-Espanha.

Neste estudo, foi encontrada associação entre composição corporal e QV, sendo notada pior QV naqueles pacientes que apresentaram déficit de massa muscular. Não foram encontrados na literatura estudos que avaliem a influência da composição corporal sobre a QV de pacientes oncológicos. Observando aspectos que podem indiretamente indicar a composição corporal (como estado nutricional e força muscular), em estudo que avaliou o impacto do estado nutricional segundo ASG e da força muscular sobre o estado de saúde geral/ $\mathrm{QV}$, utilizando a mesma amostra do presente estudo, foram observados menores escores na escala de estado geral de saúde/QV conforme piora no estado nutricional dos pacientes, já a força do aperto de mão não esteve associada à escala avaliada ${ }^{22}$.

Entende-se que novos estudos que avaliem a associação entre composiçáo corporal e QV em portadores de neoplasias malignas devam ser realizados, pois se observa uma carência na literatura de trabalhos com esse fim, sendo fundamental na terapia antineoplásica identificar e buscar o controle de aspectos que impactem na $\mathrm{QV}$ dos pacientes.

Por se tratar de um estudo com delineamento transversal, sua principal limitação refere-se à metodologia, pois esta impossibilita estabelecer relação temporal entre a composição corporal e a QV em termos de causalidade. Outra limitação do presente estudo é a utilização da BIA para avaliar a composiçáo corporal da amostra, pois se trata de um método duplamente indireto, fazendo apenas a estimativa da composição corporal.

\section{CONCLUSÃO}

Este estudo identificou déficit de massa muscular em grande parte da amostra analisada, e esta esteve associada, em todas as escalas avaliadas, à pior QV nesses pacientes, demonstrando assim que a composiçáo corporal exerce influência sobre a QV de pacientes com câncer.

Diante do exposto, percebe-se a importância da avaliação da composição corporal de pacientes oncológicos, bem como de sua $\mathrm{QV}$, pois essas informaçóes podem ser necessárias e fundamentais para as decisões de tratamento e intervenções com o objetivo de melhorar os aspectos negativos produzidos pela doença.

\section{CONTRIBUIÇÕES}

Camila Conde Frio trabalhou na concepção do estudo e redação do manuscrito. Alessandra Doumid Borges Pretto trabalhou na redaçáo do manuscrito. Maria Cristina Gonzalez trabalhou na concepção, orientação e coordenação da pesquisa. Carla Alberici Pastore trabalhou em todas as etapas da pesquisa e do manuscrito.

\section{Declaração de Conflito de Interesses: Nada a Declarar.}

\section{REFERÊNCIAS}

1. Coronha AL, Camilo ME, Ravasco P. A importância da composição corporal no doente oncológico: qual a evidência? Acta Med Port. 2011 Dec;24 Suppl 4:769-78.

2. Zandonai AP, Cardozo FMC, Gonzales Nieto IN, Sawada WO. Qualidade de vida nos pacientes oncológicos: revisão da literatura latino-americana. Rev Eletr Enf. 2010;12(3):554-61.

3. Baracos VE, Reiman T, Mourtzakis M, Gioulbasanis I, Antoum S. Body composition in patients with non-small cell lung cancer: a contemporary view of cancer cachexia with the use of computed tomography image analysis. Am J Clin Nutr. 2010 Apr;91(4):1133S-1137S.

4. Xará S, Amaral TF, Parente B. Undernutrition and quality of life in non small cell lung cancer patients. Rev Port Pneumol. 2011 Jul-Aug;17(4):153-8.

5. Toscano BAF, Coelho MS, Abreu HB, Logrado MHG, Fortes RC. Câncer: implicaçôes nutricionais. Comun Ciênc Saúde. 2008;19(2):171-80.

6. Siddiqui F, Konski AA, Movsas B. Quality-of-life concerns in lung cancer patients. Expert Rev Pharmacoecon Outcomes Res. 2010 Dec;10(6):667-76.

7. Freire MEM, Sawada NO, França ISX, Costa SFG, Oliveira CDB. Qualidade de vida relacionada à saúde de pacientes com câncer avançado: uma revisão integrativa. Rev Esc Enferm USP. 2014;48(2):357-67.

8. Nicolussi AC, Sawada N. Fatores que influenciam a qualidade de vida de pacientes com câncer de cólon e reto. Acta Paul Enferm. 2010;23 (1):125-30.

9. Gonzalez MC, Borges LB, Silveira DH, Assunção $\mathrm{MC}$, Orlandi SP. Validação da versão em português da 
avaliação subjetiva global produzida pelo paciente. Rev Bras Nutr Clin. 2010;25(2):102-8.

10. Kyle UG, Pirlich M, Lochs H, Schuetz T, Pichard C. Increased length of hospital stay in underweight and overweight patients at hospital admission: a controlled population study. Clin Nutr. 2005 Feb;24(1):133-42.

11. Ferraz LF, Campos ACF. Nutrição imunomoduladora pré-operatória em pacientes com câncer gástrico. Nutr Clín Diet Hosp. 2012;32(3):43-6.

12. Oliveira FP, Santos A, Viana MS, Alves JL, Pinho NB, Reis PF. Perfil Nutricional de pacientes com câncer de cavidade oral em pré-tratamento antineoplásico. Rev Bras Cancerol. 2015;61(3):253-59.

13. Firme LE, Gallon CW. Perfil nutricional de pacientes com carcinoma esofágico de um hospital público de Caxias do Sul. Rev Bras Cancerol. 2010;56(40):443-51.

14. Khoshnevis N, Ahmadizar F, Alizadeh M, Akbari ME. Nutritional assessment of cancer patients in Tehran, Iran. Asian Pac J Cancer Prev. 2012;13(4):1621-6

15. Campos JADB, Prado CD, Pirelli C, Garcia PPNS. Métodos de estimativa de peso e altura. Alim Nutr. 2012;23(4):681-8.

16. Trutschnigg B, Kilgour RD, Reinglas J, Rosenthall L, Hornby L, Morais JA, et al. Precision and reliability of strength (Jamar vs Biodex handgrip) and body composition (dual-energy X-ray absorptiometry vs bioimpedance analysis) measurements in advanced cancer patients. Appl Physiol Nutr Metab. 2008 Dec;33(6):1232-9.

17. Lotici T, Antunes LBB, Melhem ARF, Bennemann GD, Schiessel DL. Prevalência de perda de peso, caquexia e desnutrição em pacientes oncológicos. Reva UNIABEU. 2014;7(17):107-24.

18. Oliveira PI, Pereira CAC, Belasco AGS, Bettencourt ARC. Comparação da qualidade de vida de portadores de câncer de pulmão antes e após o tratamento quimioterápico. Rev Latino-Am Enfermagem. 2013;21(3):1-8.

19. Gupta D, Lis CG, Granick J, Grutsch JF, Vashi PG, Lammersfeld CA. Malnutrition was associated with poor quality of life in colorectal cancer: a retrospective analysis. J Clin Epidemiol. 2006 Jul;59(7):704-9.

20. Shahmoradi N, Kandiah M, Peng LS. Impact of nutritional status on the quality of life of advanced cancer patients in hospice home care. Asian Pac J Cancer Prev. 2009;10(6):1003-09.

21. Trabal J, Leyes P, Forga MT, Hervas S. Quality of life, dietary intake and nutritional status assessment in hospital admitted cancer patients. Nutr Hosp. 2006 Jul-Aug;21(4):505-10.

22. Pastore CA, Oehlschlaeger MHK, Gonzalez MC. Impacto do estado nutricional e da força muscular sobre o estado de saúde geral e qualidade de vida em pacientes com câncer de trato gastrointestinal e de pulmão. Rev Bras Cancerol. 2013;59(1):43-9. 


\section{Abstract}

Introduction: Cancer has effects on body composition, often causing sarcopenia, which impacts on the functional capacity and prognosis of these patients, which may affect their quality of life. Objective: To evaluate the influence of body composition on the quality of life of patients with cancer of the gastrointestinal tract and lungs. Method: A cross-sectional study was carried out in patients with gastrointestinal and lung cancer, in the chemotherapy service of the Hospital School of the Federal University of Pelotas. Body composition was estimated by bioelectrical impedance, performed with instrument BIA and quality of life was assessed using the European Organization for Research and Treatment of Cancer Quality of Life Questionnaire Core-30 Instrument. Results: We evaluated 74 patients, the majority of whom were male $(56.8 \%)$. The most prevalent tumors were those in the gastrointestinal tract $(75.4 \%)$. Regarding body composition, it was found that $24.3 \%$ of the patients showed a deficit in muscle mass and none had excess adiposity. When analyzing the relationship between body composition and differing scales of quality of life, patients with muscle mass deficit had lower scores on the General Health scales / QOL and functional, and higher scores on the symptoms, demonstrating worse quality of life when compared to those without muscle mass deficit. Conclusion: Body composition was related significantly to the QOL of patients with cancer of the gastrointestinal tract and lungs, keeping the association between the deficit of muscle mass and worse QOL of these patients.

Key words: Lung Neoplasms; Gastrointestinal Neoplasms; Body Composition; Quality of Life

\section{Resumen}

Introducción: El cáncer tiene efectos sobre la composición corporal, a menudo causando la sarcopenia, lo que repercute en la capacidad funcional y el pronóstico de estos pacientes, que pueden afectar su calidad de vida. Objetivo: Evaluar la influencia de la composición corporal en la calidad de vida de los pacientes con cáncer del tracto gastrointestinal y los pulmones. Método: Se realizó un estudio transversal en pacientes con cáncer gastrointestinal y pulmón, en el servicio de quimioterapia Hospital Escuela de la Universidad Federal de Pelotas. La composición corporal se calcula por impedancia bioeléctrica y la calidad de vida fue evaluada utilizando el instrumento Organización Europea para la Investigación y el Tratamiento del Cáncer Cuestionario de Calidad de Vida de 30. Resultados: Se evaluaron 74 pacientes, la mayoría del sexo masculino (56,8 \%). El tamaño de los tumores eran los más prevalentes entre el tracto gastrointestinal $(75,4 \%)$. Se encontró en la composición corporal que el 24,3\% de los pacientes presentaron un déficit en la masa muscular y ninguno presentó exceso de adiposidad. Al analizar la relación entre la composición corporal y las diferentes escalas de calidad de vida, los pacientes con déficit de masa muscular tuvieron puntuaciones más bajas en la salud general de las escalas / CDV y funcional, y puntuaciones más altas en los síntomas, lo que demuestra peor calidad de vida en comparación con los que no tienen déficit de masa muscular. Conclusión: La composición corporal con déficit de masa muscular se relaciona significativamente con la peor Calidad de Vida en los pacientes con cáncer del tracto gastrointestinal y de pulmones.

Palabras clave: Neoplasias Pulmonares; Neoplasias Gastrointestinales; Composición Corporal; Calidad de Vida 